The requirement of 1,650 patient encounters should not be dismissed as being without worth, however. Competency-based assessments are still very much in fledgling form, with educators striving to understand how to capture the data necessary for such evaluations in a manner that is accurate, reproducible, and doable. Competence requires experience so that a learner may begin to appreciate the common and not-so-common presentations of disease. The 1,650 requirement provides a surrogate marker of adequate experience to allow residency educators to begin to make an in-depth assessment of competence once adequate experience has been attained.

In our zeal to pursue competency-based assessments, it would be a grave mistake to discard all requirements based upon experience. In order to appreciate the breadth of family medicine, a resident must see a variety of patients. One does not learn all there is to know about diabetes from seeing 1 patient with diabetes. Adequate experience is key to ensuring an appreciation of the varied presentations of health and disease in patients across the spectrum of age and condition. An appreciation of the subtleties of the art of medicine cannot develop after seeing only a single example of pathology.

It would be a similarly serious error to consider the completion of 1,650 patient visits to be the sole indicator that a resident is prepared to enter practice. We ask our graduates to take on responsibility for patients, families, and communities with outstanding skills in diagnosis and treatment of disease as well as proficiency in communication, interpersonal skills, and systems-based practice. It is not enough to deem them competent after seeing a specified number of patients when what we ask of them is that they appreciate the complexity and context of each individual who presents with a given diagnosis and attend to their unique experience of health and disease in a continuous trusting relationship. A simple number can never tell us if they are prepared to undertake this critical and complex task.

Seeing 1,650 patients is a necessary but incomplete picture of a resident's preparedness to embark upon unsupervised ambulatory practice. One thousand, six hundred and fifty patient visits provide experience in which the resident can develop competence in the art of medicine. Only when experience is coupled with careful assessments of competency performed by faculty and program directors can we ensure our graduates are truly prepared for the task they undertake as family physicians.

Gretchen M. Dickson, MD, MBA, FAAFP James W. Jarvis, MD, FAAFP, Lynn Pickeral

\section{DISSEMINATION AND IMPLEMENTATION RESEARCH IN PRIMARY CARE PRACTICE- BASED RESEARCH NETWORKS}

\section{PBRN Conference Highlights}

The 2016 NAPCRG Practice-Based Research Network (PBRN) Conference brought together the energy of 196 participants from the United States, Canada, and the United Kingdom in Bethesda, Maryland on July 11-12, 2016. Conference co-chairs Rowena Dolor and L. J. Fagnan provided the welcome and orientation for this AHRQ-sponsored conference.

Rebecca Roper MS, MPH, lead scientist for the PBRN initiative at the Agency for Healthcare Research and Quality (AHRQ), gave a short overview of the centers (P30s) and the new certificate program for practice-based research methods (PBRMs). In its inaugural year (September 2015-July 2016), 16 fellows graduated from this program. For the second year, there are 54 fellows (46 mentors) from the United States and Canada enrolled. A 2-day convocation sponsored by AHRQ was held prior to the PBRN conference to evaluate the PBRM certificate program and potential modifications for the upcoming year. Roper thanked the course co-directors, James Werner, PhD, and Lyle J. Fagnan, MD, for their leadership.

Arlene Bierman, MD, Director of AHRQ's Center for Evidence and Practice Improvement, highlighted AHRQ's primary care areas of interest and achievements produced by PBRNs. She also shared some practical tools for ambulatory care clinicians, composed of a suite of point-of-care resources for the clinician team and researchers. AHRQ recognizes the critical role that PBRNs have played in creating and sustaining the viability of these tools.

Josh Tepper, MD, MPH, MBA, delivered the first plenary on "Getting to 'Better' in Ontario's Primary Care System," where he described Ontario, Canada's framework for quality and its application to primary care, the role of large scale data and reporting efforts, and the use of quality improvement (QI) plans and capacity building in QI to help improve care.

The second plenary started with the patient perspective of Vincent Dumez, MSc, on his journey developing the patient and professional partnership for primary health care collaborations. His talk was supplemented by the physician-research perspective from Antoine Boivin, MD, PhD, from Montreal, Canada. 
Together they serve as the co-principal investigators for the Quebec SUPPORT (Support for People and Patient-Oriented Research and Trials) unit in patientoriented research in primary health care. Their talk highlighted the challenges, different levels of engagement, and implementation strategies within their unit.

The third plenary was given by David Meyers, MD, Chief Medical Officer for AHRQ. He described the overarching goals of AHRQ's EvidenceNOW: Advancing Heart Health in Primary Care initiative, including the outcome measures, design elements for implementation of quality improvement strategies and research activities, and the early lessons learned.

Andrew Bindman, MD, Director of AHRQ since May 2016, emphasized the importance of primary care and PBRNs in advancing the knowledge funded and disseminated by AHRQ. He introduced Ted Ganiats, MD, who joined AHRQ in July 2016 as the Director of the National Center of Excellence in Primary Care Research who asked the audience to give him feedback on questions AHRQ has in supporting primary care research.

The 16-member PBRN planning committee reviewed 112 abstracts leading to 42 poster presentations, 10 workshops, and 48 oral presentations. Each submitter was asked to include a statement of why their research is relevant to clinical practice and patients. The 11 oral presentation tracks included Chronic Care Management, PBRN Infrastructure/ Network Operations, Practice Facilitation/Quality Improvement, Training, Dissemination/Implementation, Pediatrics/Chronic Care, Stakeholder Engagement, Behavioral Health, Chronic Care Management, Shared Decision Making, Community Engaged Research, and Technology.

The planning committee allowed for substantial time to accommodate 10 workshops. The workshop topics covered a variety of topics:

- Making composite measures of quality useful for front-line primary care providers

- Sharing research findings about how primary care and public health collaborate and an emerging model that can support integration of primary care and public health at a local level

- Using theoretical approaches in implementation and dissemination research to guide data collection and to better understand why and how implementation outcomes came about

- Best practices in survey methods for PBRNs, discussing challenges to issues such as survey feasibility and generalizability, and training in building and administering surveys using online tools

- The evolution of a community-based research network (CBRN) to create a community of solution for health care transformation
- Virtual facilitation

- Developing and maintaining PBRN learning communities which provide integrated research and quality improvement activities that carry MOC/CE for their member clinicians

- Organizing framework to help communities understand PBRN's priorities, aid in practice recruitment, and uncover areas for project development - How electronic health records (EHRs) and health information technology (HIT) can be leveraged to support large and small scale research in PBRNs

- Enacting mixed methods research in primary care

The poster sessions were well attended with ample opportunity for extended conversations and networking. Conference participants voted for the top posters to receive the David Lanier Poster Awards. Winning first place was "Identifying Primary Care Measures that Matter" (Rebecca Etz, Marshall Brooks, PhD, and Martha Gonzalez, BA). There was a 3-way tie for second place with "Does Facilitated Implementation of Shared Decision Making improve Health Outcomes for Asthma Patients? Preliminary Results from a Statewide RCT for an Asthma Dissemination Intervention" (Thomas Ludden, $\mathrm{PhD}_{i}$ Yhennko J. Taylor, $\mathrm{PhD}_{i}$ Hazel Tapp, PhD), "Co-Researching Successful Chronic Pain Management Techniques using an Appreciative Inquiry-Boot Camp Translation (AI-BCT) Process" (Mary Wold, MPH, Jodi Holtrop, PhD, MCHES; Donald Nease Jr, MD), and "Patient-Centered Research Priorities in a Pediatric PBRN" (Sarah Brewer, MPA; Sean O'Leary, MD, MPH, Natalie Crump, MS).

This year we again offered a Daring and Dangerous Ideas sessions. The concept borrowed from our primary care colleagues in the United Kingdom. In a fast-paced and interactive session, the presenter shared their dangerous PBRN research or clinical care idea that they think needs to be heard in the PBRN community. John Beasley presented, "We Should Measure and Reduce "Work After Work" (WAW)." John proposed that in the same manner that health care organizations (HCOs) are graded according to metrics related to the Triple Aim, they should be graded on metrics related to the "care of the clinician" in the Quadruple Aim. We all agreed that encouraging participants to think out of the box made for a stimulating conference.

The enthusiasm and engagement at the 2016 PBRN Conference was high from start to finish and people are excited about coming back next year. The 2017 PBRN Conference is tentatively set for June 22-23 in Bethesda with the theme of "Taking Practice Transformation to Scale With Quality Improvement." Hope to see you there next year!

Rowena Dolor, MD, MHS, and Lyle J. Fagnan, MD 